Man and Nature

L'homme et la nature

\title{
Diderot vu par ses contemporains italiens
}

\section{Rosena Davison}

Volume 5, 1986

URI : https://id.erudit.org/iderudit/1011852ar

DOI : https://doi.org/10.7202/1011852ar

Aller au sommaire du numéro

Éditeur(s)

Canadian Society for Eighteenth-Century Studies / Société canadienne d'étude du dix-huitième siècle

ISSN

0824-3298 (imprimé)

1927-8810 (numérique)

Découvrir la revue

Citer cet article

Davison, R. (1986). Diderot vu par ses contemporains italiens. Man and Nature / L'homme et la nature, 5, 63-71. https://doi.org/10.7202/1011852ar

Copyright (c) Canadian Society for Eighteenth-Century Studies / Sociéte canadienne d'étude du dix-huitième siècle, 1986
Ce document est protégé par la loi sur le droit d'auteur. L'utilisation des services d'Érudit (y compris la reproduction) est assujettie à sa politique d'utilisation que vous pouvez consulter en ligne.

https://apropos.erudit.org/fr/usagers/politique-dutilisation/ 


\section{Diderot vu par ses contemporains italiens}

Le thème de Diderot dans le contexte italien nous rappelle tout de suite l'excellent livre de Manlio Busnelli intitulé justement Diderot et l'Italie. L'auteur traite à fond la question des rapports entre Diderot et les Italiens qu'il a connus, mais vus surtout à travers les yeux de Diderot. Dans cette communication nous aimerions pour ainsi dire changer de perspective pour examiner plutôt l'image du philosophe que se sont faite ses amis ou ses connaissances italiens.

Vers la fin de son livre, Busnelli nous laisse avec l'avertissement suivant:

La grande majorité des hommes de lettres et des philosophes italiens - abstraction faite de l'abbé Galiani, de Beccaria, des frères Verri, et de quelques autres ne semble avoir eu qu'une connaissance très vague de la personnalité et de la pensée philosophique de Diderot. ${ }^{1}$

Mais ceux qui ont bien connu Diderot nous ont laissé quelques aperçus intéressants du philosophe, aussi bien de sa pensée philosophique que de sa personnalité. En examinant les mémoires de Goldoni, la correspondance des frères Verri, de Beccaria et de l'abbé Galiani, nous tenterons de reconstruire un portrait de Diderot; ce portrait, quoique partiel, complétera par des détails parfois surprenants, l'image habituelle que nous nous faisons du philosophe.

C'est en 1762 que Carlo Goldoni, le dramaturge vénitien, arrive à Paris, où il restera jusqu'à sa mort en 1793. En 1763 il débute à la 
Comédie-Italienne par une pièce intitulée L'Amour Paternel. Bachaumont écrit:

M. Goldoni, avocat de Venise et auteur d'un théâtre, s'est transporté ici pour concourir au bien-être de la Comédie-Italienne. Il travaille à présent pour elle, et ses compatriotes espèrent tirer parti de son séjour auprès d'eux. ${ }^{2}$

Goldoni rencontre Diderot dans des circonstances délicates. Diderot, accusé par Fréron dans l'Année littéraire de 1757 d'avoir emprunté largement à la comédie Il Vero Amico de Goldoni, se défend chaleureusement dans son Discours sur la poésie dramatique qui accompagne Le Père de famille paru en 1758. Au lieu de reconnaître ce qu'il devait dans l'intrigue et dans la conduite de sa pièce au dramaturge vénitien, il a préféré, selon Busnelli, 'écraser Goldoni sous le poids d'une critique hautaine et malveillante, pour exalter lui-même... les singuliers mérites de son malheureux Fils si peu naturel. ${ }^{3}$ Situation donc peu facile pour Goldoni. Il décide de réfuter les accusations contre Diderot qu'il considère 'un homme du plus grand mérite.' Dans ses Mémoires nous lisons:

Je rendis justice à M. Diderot, je tâchai de désabuser ceux qui croyaient son Père de famille puisé dans le mien; mais je ne disais rien sur le Fils naturel. L'auteur était fâché contre M. Fréron et contre moi; il voulait faire éclater son courroux, il voulait le faire tomber sur l'un ou sur l'autre, et me donna la préférence. Il fit imprimer un Discours sur la poésie dramatique, dans lequel il me traite un peu durement. ${ }^{4}$

Diderot garde rancune contre Goldoni, qui essaie en vain de se rapprocher du philosophe par l'intermédiaire d'amis communs. 'Enfin, ennuyé d'attendre, je forçai sa porte,' écrit-il. Avec son ami Duni, musicien italien qui connaissait Diderot, il s'introduit chez lui. Voici comment Goldoni décrit le déroulement de cette rencontre gênante:

M. Diderot s'efforce en vain de cacher l'embarras dans lequel mon introducteur l'avait jeté. Il ne peut pas cependant se refuser à la politesse et aux égards de la société.

On parle de choses et d'autres; la conversation tombe sur les ouvrages dramatiques. M. Diderot a la bonne foi de me dire que quelques-unes de mes pièces lui avaient causé beaucoup de chagrin; j'ai le courage de lui répondre que je m'en étais aperçu. "Vous savez, monsieur, me dit-il, ce que c'est qu'un homme blessé dans la partie la plus délicate. - Oui, monsieur, lui dis-je, je le sais; je vous entends, mais je n'ai rien à me reprocher." ${ }^{\text {s }}$ 
A ce moment, Duni intervient en leur proposant de suivre le conseil du Tasse qu'il cite en italien: ' $Q u$ 'on ne rappelle pas des souvenirs fâcheux et que tout ce qui s'est passé soit enseveli dans l'oubli.' Goldoni ajoute que Diderot 'qui entendait assez l'italien, semble souscrire de bonne grâce à l'avis du poète italien; nous finissons notre entretien par des honnêtetés, par des amitiés réciproques, et nous partons $M$. Duni et moi très contents l'un et l'autre.' ${ }^{6}$

Il me semble que le compte-rendu de cet entretien révèle deux aspects importants de la personnalité de Diderot: sa flexibilité et son amabilité; il est prêt à ensevelir sa rancune contre Goldoni une fois les malentendus dissipés, au point où Goldoni considère l'occasion 'un jour de triomphe pour moi.'

Lorsque Cesare Beccaria arrive à Paris en octobre 1766, sa renommée auprès des Encyclopédistes le précède comme auteur d'un petit livre intitulé Des Délits et des peines, publié en 1764. Disciple des philosophes, en janvier 1766 Beccaria écrit à Morellet, traducteur de son œuvre:

Les livres de d'Alembert, Diderot, Helvetius, Buffon, Hume, noms illustres que personne n'entend prononcer sans être ému, vos œuvres immortelles sont ma lecture continuelle, et l'objet de mes occupations pendant le jour et de mes méditations dans le silence de la nuit. ${ }^{7}$

Les fruits de ses méditations seront inscrits dans son livre, où il propose une réforme du code pénal, et réclame la proportion entre les délits et les peines, l'égalité dans les châtiments, la suppression de la torture et la mise en question de la légitimité de la peine de mort. Comment Diderot réagitil devant ces idées si neuves à l'époque? Nous constatons avec surprise que dans un résumé de ses Notes marginales à l'œuvre, son commentaire est plutôt négatif. Il écrit:

J'admire le fonds inépuisable d'humanité qui l'a dicté. Je révère l'auteur... cependant il s'en manque beaucoup que je croie l'ouvrage Des Délits et des peines aussi important, ni le fond des idées aussi vrai qu'on le prétend. ${ }^{8}$

Diderot réfute vivement l'argumentation de Beccaria contre la peine de mort et constate que c'est une pure illusion de considérer le salut des peuples comme la loi suprême de la société.

Ces commentaires datent de 1771 donc n'affectent en rien l'impression de Diderot que Beccaria s'est faite lors de son arrivée à Paris en 1766. Il est accompagné par le comte Alessandro Verri, qui avec son frère Pietro, avait aidé et encouragé Beccaria dans la rédaction de son œuvre. Avant 
son arrivée, Beccaria connaissait bien l'œuvre de Diderot; il avait écrit à Morellet

Ce que j'ai pu lire jusqu'à présent de $\mathrm{M}$. Diderot, c'est-à-dire ses ouvrages dramatiques, l'Interprétation de la nature et les articles de l'Encyclopédie, máa paru rempli d'idées et de chaleur. Quel excellent homme ce doit être!"9

Cette première impression favorable sera confirmée lors de leur rencontre. Le lendemain de son arrivée, Beccaria déjeune chez le baron d'Holbach; ravi, il écrit à sa femme:

Tu ne saurais croire l'accueil, les politesses, les éloges, les démonstrations d'amitié et d'estime dont mon compagnon et moi avons été comblés. Diderot, le baron $\mathrm{d}$ 'Holbach et d'Alembert se montrent particulièrement enchantés de nous... Diderot fait preuve d'enthousiasme et de bonhomie dans toutes ses manières. Tous s'efforcent à me faire plaisir, et ce sont les plus grands hommes d'Europe; tous daignent m'écouter, aucun ne montre le moindre air de supériorité. ${ }^{10}$

Malheureusement c'est la seule fois que Beccaria fait une allusion directe à Diderot dans les lettres à sa femme. Bientôt, jaloux de sa jolie femme, il repart seul pour Milan le 3 décembre 1766, laissant à Paris Alessandro Verri qui, lui, écrira plus longuement sur Diderot.

C'était plutôt son frère aîné Pietro, connu par les philosophes pour son ouvrage Sulla felicitá ou Pensées sur le bonheur, que Morellet avait invité à Paris, et non pas Alessandro. Mais celui-ci se défendit bien, et c'est dans ses lettres à Pietro que nous trouvons un portrait saisissant de Diderot, de même qu'un tableau intéressant de la société dans laquelle il se trouve présenté au même moment que Beccaria, et que son séjour prolongé lui permettra de mieux connaître que son compatriote. Voici ses premières impressions:

Le caractère qu'ils [les philosophes] exigent dans l'homme est avant tout la bonté, plutôt que la science. Entr'eux ils ont un ton tout à fait familier, philanthrope. Il n'y a pas de pompe, ni de pédanterie. Ils discutent entre eux avec chaleur et vigueur, avec toute la bonne foi du monde. ${ }^{11}$

Il poursuit en disant qu'on y mange 'divinamente,' qu'on parle beaucoup, on raisonne comme on peut, mais le ton est toujours jovial. Une semaine plus tard il s'étonnera que ces hommes de lettres puissent vivre ensemble et être amis. Il conclut que la persécution qu'ils ont soufferte a dû contribuer à les rapprocher, de même que leur bonté de cœur. Il admire également la franchise totale avec laquelle ils discutent tous les sujets, 
sans précaution et sans crainte. De tous les philosophes dont il fait la connaissance, c'est Diderot qui lui fera tracer le portrait le plus complet. Il écrit:

Diderot est la simplicité même...; il déclame toujours avec feu, il délire, il est constamment en ébulition, dans sa conversation comme dans ses livres. C'est un homme très bon, très sensible. Je lui dis en l'abordant chez le baron d'Holbach: "Je ne sais, monsieur, si je vous dois des compliments ou des adorations." Lui, avec sa simplicité habituelle, se mit tout de suite à mon niveau. "Je vois, ajoutai-je alors, que les grands philosophes sont comme les grands seigneurs: plus ils sont grands, plus ils sont humains." Il me répondit, en me prenant la main: "Je suis bien content que vous ayez cette maxime-là." ${ }^{12}$

Après une visite chez Helvétius, qu'il appelle 'un ange de bonté', Verri souligne la franchise et la simplicité de celui-ci, simplicité non seulement de manière mais aussi de tenue qui tranche curieusement avec ses talents et sa réputation, qualité qui s'applique également à Diderot et à d'Alembert. Il conclut que toutes les acclamations qu'ils ont reçues ne leur ont point fait tourner la tête. Cependant après avoir réfléchi davantage, son regard devient plus aigu et il critique la façon de raisonner des philosophes; de Londres en janvier 1767 il écrit à Pietro:

La chaleur avec laquelle ils écrivent annonce la vérité, mais si vous observez bien, si vous analysez et pesez leurs idées, vous verrez qu'il y a des cascades à côté du sublime. ${ }^{13}$

En 1770 Verri écrit à son frère que Diderot est partisan de Galiani, et qu'il estime beaucoup son livre. Ce Galiani est l'abbé Ferdinando Galiani, grand ami de Diderot, et le livre en question est les Dialogues sur le commerce des blés que Galiani avait laissés entre les mains de Diderot pour éditer et publier après le retour précipité de l'abbé à Naples en 1769. Galiani avait passé une dizaine d'années dans la capitale et s'était lié avec les philosophes, surtout avec Diderot. Parmi tous les Italiens qui avaient rencontré Diderot, Galiani est sans doute celui qui le connaissait le mieux: son témoignage nous est donc parmi les plus intéressants. Très tôt après son départ, Galiani se plaint amèrement que Diderot est 'le plus mauvais de tous les correspondants'; mais lui-même il ne fait pas mieux; il le reconnaît et s'excuse en écrivant ainsi à Mme d'Epinay: 'Je suis honteux de n'avoir pas encore répondu à Diderot; mais comme le philosophe ne connaît pas la durée du temps, il n'y aura ni tôt ni tard pour lui. ${ }^{14} \mathrm{Ce}$ sera donc Mme d'Epinay qui agira comme intermédiaire entre ces deux mauvais correspondants, et qui les tiendra au courant de 
leurs activités respectives. Mais lorsque Diderot prend lui-même la plume, c'est une effusion de joie et de sentiment de la part de Galiani; celui-ci lui répond: 'Je reçois quelques lignes de vous, qui ne me paraissent précieuses que par l'écriture et la main qui les a tracées.' Il termine sa lettre en disant: 'Je vous embrasse, cher philosophe, de tout mon cœur. Aimez celui qui vous adore. ${ }^{\prime 15}$

Galiani avoue que c'est surtout lorsqu'il va au théâtre à Naples que l'absence de Diderot lui 'trouble tout le plaisir du spectacle.' Au début de 1773 Galiani aura le plaisir d'assister à une série de pièces françaises montées à Naples par une troupe française. Il s'empresse d'en rendre compte à Mme d'Epinay, en lui demandant d'en faire part à Diderot:

Ils ont débuté par la pièce du Père de famille, parce que c'est, de toutes les pièces de théâtre français, celle dont le succès est le plus grand et le plus assuré dans toutes les villes d'Italie et d'Allemagne... Mes Napolitains sont convaincus que sa pièce est la meilleure de tout le théâtre français, et par conséquent la meilleure production dramatique de l'esprit humain jusqu'à cette heure. ${ }^{16}$

Ces éloges sont très forts, et d'autant plus frappants qu'en 1764 Galiani avait écrit dans un de ses rapports confidentiels régulièrement adressés à Tanucci, ministre des affaires étrangères à Naples, que Diderot avait écrit d'excellents traités sur le drame, et qu'il avait écrit deux comédies médiocres. Dans ce même rapport, Galiani explique que Diderot, chef de l'entreprise encyclopédique, est celui parmi tous les Français de sa connaissance, qui connaît le mieux les différentes littératures, et que la passion dominante chez lui est la métaphysique. Ce que Galiani respecte le plus chez celui qu'il appelle 'notre grand Diderot' est non seulement ses idées mais également sa façon de s'exprimer. Par exemple, à propos de son commentaire sur le Dialogue sur les femmes de Thomas que Mme d'Epinay lui avait envoyé et auquel Galiani a essayé de répondre, Galiani écrit:

Je vous remercie de la feuille de Diderot. Elle est digne de lui, et ne ressemble en rien à mon dialogue, mais il est écrit à côté des dames parisiennes, et moi j'écris à côté des femmes napolitaines. Il trempe sa plume dans l'arc-en-ciel et je la trempe dans la thériaque. Son écrit ressemble à un paon; le mien à un chauve-souris. ${ }^{17}$

A une autre occasion, c'est l'Eloge de Rouelle, le grand chimiste, que Galiani admire; tout en le qualifiant de 'chef-d'œuvre' de Diderot, il poursuit:

Mais ce diable de Diderot est d'une véracité qui incommode peut-être même les 
morts. Au reste, il est étonnant combien, à force de paroles, il dessine, colorie, anime ses tableaux. Je crois qu'on a connu Rouelle, quand on a lu ce portrait. ${ }^{18}$

Dans les lettres de Galiani on trouve un curieux mélange de respect, d'amitié et d'étonnement au sujet du philosophe qu'il avait si bien connu à Paris. D'une part il déclare que 'Diderot ne sait rien de ce qui se passe dans la nature, malgré qu'il en ait interprété les secrets, ${ }^{\prime 19}$ et d'autre part que Diderot ne veut pas 'se guérir de la manie de faire du bien aux hommes,' parce que la corvée du sage est de faire du bien aux hommes. ${ }^{20}$

A Naples, Diderot lui manque beaucoup, et sur plusieurs plans. Lorsque Grimm lui rend visite en 1776 Galiani écrit: 'Je ne lui ai parlé que du philosophe, lorsque je pensais à m'égayer.'21 Il regrette les échanges, les conversations qu'il avait tant aimés avec Diderot. Le courrier et les bons offices de Mme d'Epinay leur permettent de se consulter de temps en temps sur des sujets aussi variés que l'interprétation d'une ode d'Horace, la nature de l'électricité, le manuscrit des Dialogues et des questions relatives à l'exportation du blé, l'inscription pour la statue que Catherine II voulait ériger pour Pierre le Grand, etc.

Lorsque Diderot décide de se rendre en Russie en 1773 en passant par la Hollande, Galiani, inquiet, craint pour son retour. Il écrit à Mme d'Epinay:

S'il allait imiter Descartes? Si les caresses d'une souveraine philosophe allaient le retenir! et puis c'est un homme à oublier qu'il doit revenir; le temps et l'espace sont devant lui comme devant Dieu: il croit être partout et être éternel. ${ }^{22}$

Une semaine plus tard, il écrit:

Je crains pour Diderot: il va trop au nord. Un voyage si pénible, au milieu des armées; c'est bien fou ce qu'il fait. ${ }^{23}$

Deux mois plus tard, c'est le même thème:

Le philosophe, à la Haye, électrisera les tortues hollandaises. Il ira en Russie, je n'en doute pas, ou pour mieux dire, il se trouvera à Pétersbourg un beau matin, sans savoir comment il y est parvenu.

Ce qu'il conçoit comme la distraction de Diderot est un thème constant dans les lettres de Galiani. Une fois rendu à Pétersbourg, c'est le comportement du philosophe avec Catherine qui le choque:

On débite sur sa conduite, près de la czarine, des choses épouvantables. On dit 
qu'il a osé lui jeter sa perruque au nez, lui pincer le genou, etc. Il est unique, ce Diderot; sa tête est le magasin du monde; il sait tout, et paraît quelquefois ne rien savoir. Je crois qu'il a, pour le moins, autant d'absences d'esprit et de raison que Thomas, ou la plupart des grands hommes de Bicêtre. ${ }^{25}$

La preuve que Galiani connaît à fond l'esprit de son ami parisien se trouve dans sa façon d'interpréter le symbolisme des objets-souvenirs que Diderot rapporte de son voyage en Russie. Il écrit:

Ils [Diderot et Grimm] arrivent précédés de squelettes, de dominos et de pantoufles. Quelle profondeur! quelle sublimité! J'entends. Le philosophe dit par le domino, que le monde n'est qu'une mascarade; par le squelette, que la mort démasque tout, et par la pantoufle, qu'il n'y a de vrai, de solide, de sérieux dans le monde, qu'une jolie pantoufle d'une jolie femme. ${ }^{26}$

Cet ami napolitain a pu cerner mieux que ses compatriotes les 'mystères' de l'esprit du philosophe, de même que ses préoccupations principales.

A Paris, Diderot a connu plusieurs Italiens, dont quelques-uns étaient simplement de passage comme Beccaria ou Verri, et d'autres qui avaient choisi la capitale comme lieu de résidence, comme Goldoni et Galiani. Chacun a apporté à Diderot les richesses de son pays, que le philosophe appréciait tant, et chacun a été frappé par un aspect différent de la personnalité de Diderot. Le nombre d'Italiens choisis pour cette étude est nécessairement restreint mais les lettres et les mémoires que nous avons consultés révèlent un Diderot parfois poli et aimable, parfois modeste, parfois distrait, mais toujours brillant, érudit et impulsif.

\section{ROSENA DAVISON}

Simon Fraser University

\section{NOTES}

1 Manlio Busnelli, Diderot et l'Italie (Paris: Champion 1925), 259

2 Cité par Georges Roth dans Denis Diderot, Correspondance. Recueillie, établie et annotée par Georges Roth et Jean Varloot. 16 vols. (Paris: Editions de Minuit 1955-70), IV, 87

3 Busnelli, 134 
4 Carlo Goldoni, Mémoires de M. Goldoni pour servir à l'histoire de sa vie et à celle de son théâtre, édition présentée et annotée par Paul de Roux (Paris: Mercure de France 1965), 301-2

5 lbid., 302

6 Ibid.

7 Cité par Busnelli, 249

8 Diderot, Oeuvres complètes éditées par Assézat et Tourneux, 20 vols, (Paris: Garnier 1875-77), IV, 61

9 Cité par Franco Venturi, Illuministi Italiani (Milan: Ricciardi 1958), III, 202.

10 Cesare Beccaria, Opere (Florence: Sansoni 1958), 882

11 Carteggio di Pietro e Alessandro Verri, a cura di Gianmarco Gaspari, (Milan: Adelphi 1980), 23

12 Ibid., 23-4

13 Ibid., 210

14 Lettre du 8 septembre 1770. Correspondance de l'abbé Galiani avec Mme d'Epinay etc. éditée par Lucien Perey et Gaston Maugras. 2 Vols. (Paris: Calmann Lévy 1881), I, 256

15 Ibid., II, 108

16 Ibid., 158-9

17 Lettre du 25 avril 1772, ibid., 63-4

18 Lettre du 7 septembre 1771, ibid., I, 443

19 Lettre du 14 juin 1777, ibid., II, 515-6

20 Lettre du 24 février 1772, ibid., I, 72

21 Lettre du 1 juin 1776, ibid., II, 444

22 Lettre du 15 mai 1773, ibid., 204

23 Ibid., 208

24 Ibid., 231

25 Lettres de l'abbé Galiani à Mme d'Epinay etc., éditées par Eugène Asse. 2 vols. (Paris: Charpentier 1881), I, 401

26 Lettre du 29 octobre 1774, Correspondance de l'abbé Galiani, éd. Perey et Maugras, II, 363 